\title{
WRONSKIANS AND LINEAR INDEPENDENCE
}

\author{
ALIN BOSTAN AND PHILIPPE DUMAS
}

Abstract. We give a new and simple proof of the fact that a finite family of analytic functions has a zero Wronskian only if it is linearly dependent.

The Wronskian of a finite family $f_{1}, \ldots, f_{n}$ of $(n-1)$-times differentiable functions is defined as the determinant $\mathrm{W}\left(f_{1}, \ldots, f_{n}\right)$ of the Wronskian matrix

$$
\left[\begin{array}{ccc}
f_{1} & \cdots & f_{n} \\
f_{1}^{\prime} & \cdots & f_{n}^{\prime} \\
\vdots & \vdots & \vdots \\
f_{1}^{(n-1)} & \cdots & f_{n}^{(n-1)}
\end{array}\right] .
$$

Obviously, a family of linearly dependent functions has a zero Wronskian.

Many standard textbooks on differential equations (e.g., [10, Chap. 5, §5.2], [22, Chap. 1, §4], [9, Chap. 3, §7]) contain the following warning: linearly independent functions may have an identically zero Wronskian! This seems to have been pointed out for the first time by Peano [20,21], who gave the example of the pair of functions $f_{1}(x)=x^{2}$ and $f_{2}(x)=x|x|$ defined on $\mathbb{R}$, which are linearly independent but whose Wronskian vanishes. Subsequently, Bôcher [1] showed that there even exist families of infinitely differentiable real functions sharing the same property. However, it is known that under some regularity assumptions, the identical vanishing of the Wronskian does imply linear dependence. The most important result in this direction is the following.

Theorem 1. A finite family of linearly independent (real or complex) analytic functions has a nonzero Wronskian.

Although this property is classical, the only direct proof that we have been able to find in the literature is that of Bôcher [2, pp. 91-92]. It proceeds by induction on the number of functions, and thus it is not very "transparent".

In most references, Theorem 1 is usually presented as a consequence of the more general fact that if the Wronskian of a family of real functions is zero on an interval, then there exists a subinterval on which the family is linearly dependent. The latter result is also proved by induction, in one of the following ways: either directly using a recursive property of the $\operatorname{Wronskian}^{1}$ (see, e.g., [13, Theorem 3]) or indirectly, making use of Bôcher's criterion [3, Theorem II]; see also [9, Chap. 3, $\S 7]$ for a simplified proof.

In the nonanalytic case, Bôcher [3] and Curtiss [5] (among others) have given various sufficient conditions to guarantee results similar to Theorem 1; some of them are recalled in [13]. In the analytic case, the property is purely formal; as a

\footnotetext{
${ }^{1}$ The idea of this proof goes back to $[7, \S 1]$; quite paradoxically, Frobenius failed to add the word "subinterval" in his original paper, and this lapse was at the origin of Peano's warnings.
} 
consequence, we use formal power series instead of functions, and we give a new, simple proof of the following extension of Theorem 1.

Theorem 2. Let $\mathbb{K}$ be a field of characteristic zero. A finite family of formal power series in $\mathbb{K}[[x]]$, or rational functions in $\mathbb{K}(x)$, has a zero Wronskian only if it is linearly dependent over $\mathbb{K}$.

Theorem 2 is used for instance by Newman and Slater in their study [18] of Waring's problem for the ring of polynomials. Note that the assumption on the characteristic is important; if $p$ is a prime number, the polynomials 1 and $x^{p}$ are linearly independent over $\mathbb{K}=\mathbb{Z} / p \mathbb{Z}$, but have zero Wronskian. Nevertheless, a variant of Theorem 2 still holds [11, Theorem 3.7] provided linear dependence over $\mathbb{K}$ is replaced by linear dependence over the ring of constants $\mathbb{K}\left[\left[x^{p}\right]\right]$ (or over the field of constants $\mathbb{K}\left(x^{p}\right)$ in the statement for rational functions).

Theorem 2 is proved for polynomials in [14, Theorem 4.7(a)]. It is a particular case of [11, Theorem 3.7]; see also [15, Prop. 2.8]. As expected, the proofs in [14, $11,15]$ are slight variations of Bôcher's inductive proof mentioned above. We now present a different proof, which is direct and effective, of Theorem 2. Our proof also has the advantage that it generalizes to the multivariate case, as we show below.

Wronskians of monomials. The key to our proof is the following classical result, which relates the Wronskian of a family of monomials $x^{d_{1}}, \ldots, x^{d_{n}}$ to the Vandermonde determinant

$$
\mathrm{V}\left(d_{1}, \ldots, d_{n}\right)=\left|\begin{array}{ccc}
1 & \cdots & 1 \\
d_{1} & \cdots & d_{n} \\
\vdots & \vdots & \vdots \\
d_{1}^{n-1} & \cdots & d_{n}^{n-1}
\end{array}\right|=\prod_{1 \leq i<j \leq n}\left(d_{j}-d_{i}\right)
$$

associated to their exponents; see, e.g., [15, Example 2.3] and [12, Theorem 24].

Lemma 1. The Wronskian of the monomials $a_{1} x^{d_{1}}, \ldots, a_{n} x^{d_{n}}$ is equal to

$$
\mathrm{V}\left(d_{1}, \ldots, d_{n}\right) x^{d_{1}+\cdots+d_{n}-\left(\begin{array}{c}
n \\
2
\end{array}\right)} \prod_{i=1}^{n} a_{i} .
$$

Proof. By definition, the Wronskian $\mathrm{W}\left(a_{1} x^{d_{1}}, \ldots, a_{n} x^{d_{n}}\right)$ is equal to the determinant of the matrix

$$
\left[\begin{array}{ccc}
a_{1} x^{d_{1}} & \cdots & a_{n} x^{d_{n}} \\
a_{1} d_{1} x^{d_{1}-1} & \cdots & a_{n} d_{n} x^{d_{n}-1} \\
\vdots & \vdots & \vdots \\
a_{1}\left(d_{1}\right)_{n-1} x^{d_{1}-(n-1)} & \cdots & a_{n}\left(d_{n}\right)_{n-1} x^{d_{n}-(n-1)}
\end{array}\right],
$$

where $(d)_{k}$ denotes the falling factorial $d(d-1) \cdots(d-k+1)$.

This determinant is equal to the product of the monomial $a_{1} \cdots a_{n} \cdot x^{d_{1}+\cdots+d_{n}-\left(\begin{array}{c}n \\ 2\end{array}\right)}$ and the determinant of the matrix

$$
D=\left[\begin{array}{ccc}
1 & \cdots & 1 \\
d_{1} & \cdots & d_{n} \\
\left(d_{1}\right)_{2} & \cdots & \left(d_{n}\right)_{2} \\
\vdots & \vdots & \vdots \\
\left(d_{1}\right)_{n-1} & \cdots & \left(d_{n}\right)_{n-1}
\end{array}\right]
$$


Since $(d)_{k}$ is a monic polynomial of degree $k$ in $d$, we can use elementary column operations (which preserve the determinant) to transform the matrix $D$ into the Vandermonde matrix associated to $d_{1}, \ldots, d_{n}$. The result follows.

Reduction to power series with distinct orders. The next result relates the Wronskian of a linearly independent family of power series and the Wronskian of a family of power series having mutually distinct orders. Recall that the order of a nonzero power series is the smallest exponent with nonzero coefficient in that series.

Lemma 2. Let $\mathbb{K}$ be a field and let $f_{1}, \ldots, f_{n}$ be a family of power series in $\mathbb{K}[[x]]$ which are linearly independent over $\mathbb{K}$. There exists an invertible $n \times n$ matrix $A$ with entries in $\mathbb{K}$ such that the power series $g_{1}, \ldots, g_{n}$ defined by

$$
\left[\begin{array}{lll}
g_{1} & \cdots & g_{n}
\end{array}\right]=\left[\begin{array}{lll}
f_{1} & \cdots & f_{n}
\end{array}\right] \cdot A
$$

are all nonzero and have mutually distinct orders. As a consequence, the following equality holds

$$
\mathrm{W}\left(g_{1}, \ldots, g_{n}\right)=\mathrm{W}\left(f_{1}, \ldots, f_{n}\right) \cdot \operatorname{det}(A) .
$$

Proof. If two series $f_{1}$ and $f_{2}$ are linearly independent, then, up to reindexing, an appropriate linear combination of $f_{1}$ and $f_{2}$ yields a nonzero series $\tilde{f}_{2}$ with order strictly greater than the order of $f_{1}$. Using this idea repeatedly proves the existence of the matrix $A$. The whole procedure can be interpreted as Gaussian elimination by elementary column operations, which computes the column echelon form of the (full rank) matrix with $n$ columns and an infinite number of rows whose columns contain the coefficients of the power series $f_{1}, \ldots, f_{n}$. The matrix $A$ in equation (1) is then equal to a product of elementary matrices, and it is thus invertible. By successive differentiations, equation (1) implies

$$
\left[\begin{array}{lll}
g_{1}^{(i)} & \cdots & g_{n}^{(i)}
\end{array}\right]=\left[\begin{array}{lll}
f_{1}^{(i)} & \cdots & f_{n}^{(i)}
\end{array}\right] \cdot A \quad \text { for all } i \geq 1,
$$

from which equation (2) follows straightforwardly.

From power series with distinct orders to monomials. For a nonzero power series $f$ in $\mathbb{K}[[x]]$, we denote by $\operatorname{LM}(f)$ the leading monomial of $f$, that is, the monomial of the smallest order among the terms of $f$ :

$$
f=\mathrm{LM}(f)+\text { (terms of higher order). }
$$

Lemma 3. Let $\mathbb{K}$ be a field of characteristic zero. If the nonzero series $g_{1}, \ldots, g_{n}$ in $\mathbb{K}[[x]]$ have mutually distinct orders, then their Wronskian $\mathrm{W}\left(g_{1}, \ldots, g_{n}\right)$ is nonzero.

Proof. If the $g_{i}$ 's are all monomials, the result is a direct consequence of Lemma 1. Indeed, the Vandermonde determinant $\mathrm{V}\left(d_{1}, \ldots, d_{n}\right)$ is nonzero if and only if the $d_{i}$ 's are mutually distinct. In the general case, let $\operatorname{LM}\left(g_{j}\right)=a_{j} x^{d_{j}}$ be the leading monomial of $g_{j}$. Then the $(i, j)$ entry of the Wronskian matrix, which was $w_{i, j}=$ $a_{j}\left(d_{j}\right)_{i-1} x^{d_{j}-i+1}$ in Lemma 1 , now becomes $w_{i, j} \times\left(1+x r_{i, j}\right)$ for some power series $r_{i, j}$ in $\mathbb{K}[[x]]$. The matrix $D$ in the proof of Lemma 1 is replaced by a matrix whose $(i, j)$ entry is

$$
\left(d_{j}\right)_{i-1} \times\left[1+x r_{i, j}\right] .
$$

The determinant of this new matrix $D$ is nonzero, since it is nonzero modulo $x$. 
Proof of Theorem 2. Let $f_{1}, \ldots, f_{n}$ be linearly independent power series in $\mathbb{K}[[x]]$. According to Lemma 2, there exist power series $g_{1}, \ldots, g_{n}$ with mutually distinct orders such that the Wronskians $\mathrm{W}\left(f_{1}, \ldots, f_{n}\right)$ and $\mathrm{W}\left(g_{1}, \ldots, g_{n}\right)$ are equal up to a nonzero multiplicative factor in $\mathbb{K}$. By Lemma 3 , the Wronskian $\mathrm{W}\left(g_{1}, \ldots, g_{n}\right)$ is nonzero; therefore the Wronskian $\mathrm{W}\left(f_{1}, \ldots, f_{n}\right)$ is nonzero as well.

If now the $f_{i}$ 's are linearly independent rational functions in $\mathbb{K}(x)$, then we can view them as Laurent series, and apply (a slight extension of) the preceding result for power series. Alternatively, one could perform a translation of the variable which ensures that the origin is not a pole of any of the $f_{i}$ 's, and then appeal to the result in $\mathbb{K}[[x]]$. In both cases, $\mathrm{W}\left(f_{1}, \ldots, f_{n}\right)$ is nonzero.

Generalized Wronskians. The concept of generalized Wronskians was introduced by Ostrowski [19] and used by Dyson [6] and Roth [23] in the context of the Thue-Siegel-Roth theorem on irrationality measures for algebraic numbers.

Let $\Delta_{0}, \ldots, \Delta_{n-1}$ be differential operators of the form (using the notation of [24, Chap. $5, \S 9],[4$, Chap. $6, \S 5],[14, \S 4-3],[8, \S$ D.6], [16, Chap. 5, §3], and [17, §6.4])

$$
\Delta_{s}=\left(\frac{\partial}{\partial x_{1}}\right)^{j_{1}} \cdots\left(\frac{\partial}{\partial x_{m}}\right)^{j_{m}} \text { with } \quad j_{1}+\cdots+j_{m} \leq s .
$$

The generalized Wronskian associated to $\Delta_{0}, \ldots, \Delta_{n-1}$ of a family $f_{1}, \ldots, f_{n}$ of power series in $\mathbb{K}\left[\left[x_{1}, \ldots, x_{m}\right]\right]$ is defined as the determinant of the matrix

$$
\left[\begin{array}{ccc}
\Delta_{0}\left(f_{1}\right) & \cdots & \Delta_{0}\left(f_{n}\right) \\
\Delta_{1}\left(f_{1}\right) & \cdots & \Delta_{1}\left(f_{n}\right) \\
\vdots & \vdots & \vdots \\
\Delta_{n-1}\left(f_{1}\right) & \cdots & \Delta_{n-1}\left(f_{n}\right)
\end{array}\right] .
$$

Obviously, there are finitely many generalized Wronskians constructed in this way. Using the same ideas as above, one can prove the following generalization of Theorem 2:

Theorem 3. If $\mathbb{K}$ has characteristic zero and if the power series $f_{1}, \ldots, f_{n}$ in $\mathbb{K}\left[\left[x_{1}, \ldots, x_{m}\right]\right]$ are linearly independent over $\mathbb{K}$, then at least one of the generalized Wronskians of $f_{1}, \ldots, f_{n}$ is not identically zero.

Two kinds of proofs of this result were previously available: one by substitution and reduction to the univariate case, in [14, Theorem 4.7(b)], [16, Chap. 5, §5], and [17, Lemma 6.4.6], the other by induction, in [4, Chap. 6, Lemma 6], [24, Chap. 5, Lemma 9A], and [8, Lemma D.6.1]. To the best of our knowledge, the following proof is new. It essentially reduces the study of Theorem 3 to the particular case when all the $f_{i}$ 's are monomials, and then concludes by using an "effective" argument in that case.

Proof. We will mimic the proof given above for the univariate case. Much as in that case (Lemma 2), the linear independence of the power series $f_{1}, \ldots, f_{n}$ implies the existence of an invertible matrix $A$ as in Lemma 2, yielding series $g_{1}, \ldots, g_{n}$ whose leading monomials have mutually distinct exponents. Here, by exponent of a nonzero monomial $c \cdot x_{1}{ }^{\alpha_{1}} \cdots x_{m}{ }^{\alpha_{m}}(c \in \mathbb{K})$ we mean the multi-index $\left(\alpha_{1}, \ldots, \alpha_{m}\right)$ in $\mathbb{N}^{m}$, and by leading monomial of a power series $f$ in $\mathbb{K}\left[\left[x_{1}, \ldots, x_{m}\right]\right]$, we mean the minimal nonzero monomial of $f$ with respect to the lexicographic order on the exponents of monomials from $\mathbb{K}\left[x_{1}, \ldots, x_{m}\right]$. 
The leading monomial of a generalized Wronskian $W$ of $g_{1}, \ldots, g_{n}$ is equal to the corresponding generalized Wronskian $W_{0}$ of their leading monomials, provided that $W_{0}$ is nonzero. Indeed, by the multilinearity of the determinant, $W$ can be written as the sum of $W_{0}$ and $2^{n}-1$ generalized Wronskians related to the same differential operators. The lexicographic order being compatible with the partial derivatives and the product of monomials, $W_{0}$ is smaller than all the monomials occuring in the other $2^{n}-1$ generalized Wronskians.

We can therefore assume from now on that the $g_{i}$ 's are all nonzero monomials:

$$
g_{i}=c_{i} \cdot \boldsymbol{x}^{\boldsymbol{\alpha}_{i}}=c_{i} \cdot x_{1}^{\alpha_{i, 1}} \cdots x_{m}^{\alpha_{i, m}}, \quad \text { for } \quad 1 \leq i \leq n,
$$

with mutually distinct exponents $\boldsymbol{\alpha}_{i}=\left(\alpha_{i, 1}, \ldots, \alpha_{i, m}\right)$. The generalized Wronskian of $g_{1}, \ldots, g_{n}$, associated to $\Delta_{0}, \ldots, \Delta_{n-1}$ of the form (3), is then equal to a nonzero monomial times the determinant of the matrix

$$
\left[\begin{array}{ccc}
1 & \cdots & 1 \\
\delta_{1}\left(\boldsymbol{\alpha}_{1}\right) & \cdots & \delta_{1}\left(\boldsymbol{\alpha}_{n}\right) \\
\vdots & \vdots & \vdots \\
\delta_{n-1}\left(\boldsymbol{\alpha}_{1}\right) & \cdots & \delta_{n-1}\left(\boldsymbol{\alpha}_{n}\right)
\end{array}\right]
$$

where, for $\boldsymbol{\alpha}=\left(\alpha_{1}, \ldots, \alpha_{m}\right)$ and $\boldsymbol{j}=\left(j_{1}, \ldots, j_{m}\right)$, we set

$$
\delta_{s}(\boldsymbol{\alpha})=(\boldsymbol{\alpha})_{j}=\left(\alpha_{1}\right)_{j_{1}} \cdots\left(\alpha_{m}\right)_{j_{m}}, \quad \text { with } \quad j_{1}+\cdots+j_{m} \leq s .
$$

Let us suppose by contradiction that all these generalized Wronskians are zero. Consider the Vandermonde determinant $\varphi$ in $\mathbb{K}\left[\left(u_{i}\right),\left(\alpha_{i, j}\right)\right]$ of the family

$$
u_{1} \alpha_{1,1}+\cdots+u_{m} \alpha_{1, m} \quad \ldots \quad u_{1} \alpha_{n, 1}+\cdots+u_{m} \alpha_{n, m},
$$

where the $u_{i}$ 's are new indeterminates. Then $\varphi$ is seen to be a homogeneous polynomial in $u_{1}, \ldots, u_{m}$, whose coefficients are $\mathbb{K}$-linear combinations of generalized Vandermonde determinants of the form

$$
\left[\begin{array}{ccc}
1 & \cdots & 1 \\
\boldsymbol{\alpha}_{1} \boldsymbol{j}_{1} & \cdots & \boldsymbol{\alpha}_{n}^{\boldsymbol{j}_{1}} \\
\vdots & \vdots & \vdots \\
\boldsymbol{\alpha}_{1} \boldsymbol{j}_{n-1} & \cdots & \boldsymbol{\alpha}_{n} \boldsymbol{j}_{n-1}
\end{array}\right], \text { with } \quad\left|\boldsymbol{j}_{s}\right| \leq s \quad \text { for } \quad 1 \leq s \leq n-1 .
$$

Here, for $\boldsymbol{\alpha}=\left(\alpha_{1}, \ldots, \alpha_{m}\right)$ and $\boldsymbol{j}=\left(j_{1}, \ldots, j_{m}\right)$, we use the classical notations $|\boldsymbol{j}|=j_{1}+\cdots+j_{m}$ and $\boldsymbol{\alpha}^{\boldsymbol{j}}=\alpha_{1}{ }^{j_{1}} \cdots \alpha_{m}^{j_{m}}$.

Each of these generalized Vandermonde determinants, and thus also $\varphi$ itself, is a $\mathbb{K}$-linear combination of determinants of the form (4). By assumption, this yields $\varphi=0$, which in turn implies, by the classical theorem on Vandermonde determinants, that there exist $i \neq j$ such that

$$
u_{1} \alpha_{i, 1}+\cdots+u_{m} \alpha_{i, m}=u_{1} \alpha_{j, 1}+\cdots+u_{m} \alpha_{j, m}
$$

Hence $\boldsymbol{\alpha}_{i}=\boldsymbol{\alpha}_{j}$, and this contradicts the hypothesis that the exponents $\boldsymbol{\alpha}_{i}$ are mutually distinct.

Acknowledgments. We thank the three referees for their useful remarks. This work has been supported in part by the Microsoft Research-INRIA Joint Centre. 


\section{REFERENCES}

[1] M. Bôcher, On linear dependence of functions of one variable, Bull. Amer. Math. Soc. 7 (1900) 120-121.

[2] - The theory of linear dependence, Ann. of Math. (2) $2(1900 / 01)$ 81-96.

[3] Certain cases in which the vanishing of the Wronskian is a sufficient condition for linear dependence, Trans. Amer. Math. Soc. 2 (1901) 139-149.

[4] J. W. S. Cassels, An Introduction to Diophantine Approximation, Cambridge Tracts in Mathematics and Mathematical Physics, No. 45, Cambridge University Press, New York, 1957.

[5] D. R. Curtiss, The vanishing of the Wronskian and the problem of linear dependence, Math. Ann. 65 (1908) 282-298.

[6] F. J. Dyson, The approximation to algebraic numbers by rationals, Acta Math. 79 (1947) 225-240.

[7] G. Frobenius, Ueber die Determinante mehrerer Functionen einer Variabeln, J. Reine Angew. Math. 77 (1874) 245-257.

[8] M. Hindry and J. H. Silverman, Diophantine Geometry, Graduate Texts in Mathematics, vol. 201, Springer-Verlag, New York, 2000.

[9] W. Hurewicz, Lectures on Ordinary Differential Equations, Technology Press of the Massachusetts Institute of Technology, Cambridge, MA, 1958.

[10] E. L. Ince, Ordinary Differential Equations, Dover, New York, 1944.

[11] I. Kaplansky, An Introduction to Differential Algebra, 2nd ed., Hermann, Paris, 1976.

[12] A. V. Kiselev, Associative homotopy Lie algebras and Wronskians, J. Math. Sci. 141 (2007) $1016-1030$.

[13] M. Krusemeyer, The teaching of mathematics: Why does the Wronskian work? This Monthly 95 (1988) 46-49.

[14] W. J. LeVeque, Topics in Number Theory. Vol. II, Dover, Mineola, NY, 2002.

[15] A. R. Magid, Lectures on Differential Galois Theory, University Lecture Series, vol. 7, American Mathematical Society, Providence, RI, 1994.

[16] K. Mahler, Lectures on Diophantine Approximations. Part I: g-adic Numbers and Roth's Theorem, University of Notre Dame Press, Notre Dame, IN, 1961.

[17] S. J. Miller and R. Takloo-Bighash, An Invitation to Modern Number Theory, Princeton University Press, Princeton, NJ, 2006.

[18] D. J. Newman and M. L. Slater, Waring's problem for the ring of polynomials, J. Number Theory 11 (1979) 477-487.

[19] A. Ostrowski, Über ein Analogon der Wronskischen Determinante bei Funktionen mehrerer Veränderlicher, Math. Z. 4 (1919) 223-230.

[20] G. Peano, Sur le déterminant Wronskien, Mathesis 9 (1889) 75-76.

[21] Sur les Wronskiens, Mathesis 9 (1889) 110-112.

[22] E. G. C. Poole, Introduction to the Theory of Linear Differential Equations, Dover, New York, 1960.

[23] K. F. Roth, Rational approximations to algebraic numbers, Mathematika 2 (1955) 1-20; corrigendum, 168.

[24] W. M. Schmidt, Diophantine Approximation, Lecture Notes in Mathematics, vol. 785, Springer, Berlin, 1980.

Algorithms Project, Inria Paris-Rocquencourt, 78153 Le Chesnay, France

E-mail address: Alin.Bostan@inria.fr

Algorithms Project, Inria Paris-Rocquencourt, 78153 Le Chesnay, France

E-mail address: Philippe.Dumas@inria.fr 